\title{
The Knowledge Exchange-Decision Support Model: application to cancer navigation programs
}

\author{
A. Fuchsia Howard • Kirsten Smillie • Vivian Chan • \\ Sandra Cook • Arminee Kazanjian
}

Received: 11 January 2013 / Accepted: 11 September 2013 /Published online: 26 September 2013

(C) The Author(s) 2013. This article is published with open access at Springerlink.com

\begin{abstract}
Purpose The Knowledge Exchange-Decision Support (KEDS) Model provides a framework outlining essential components of knowledge generation and exchange. The purpose of this research was to illustrate how the Model makes explicit the different contextual aspects implicit in the planning and implementation of two cancer navigation programs in Canada.

Methods The KE-DS Model guided the collection and analysis of interviews with program personnel and narrative data. A qualitative thematic analysis was conducted wherein we compared and contrasted the planning and implementation of these two navigation programs.

Results The planning and implementation of these two programs was conceptualized differently and adapted to meet local contingencies. The KE-DS Model highlighted three factors that influenced program delivery. First, the structure of health services was shaped by the interaction of professionals and services operating in the region, and the existing health services influenced the program's approach to navigation. Second, while there were similarities in the professional roles and responsibilities of the navigators, these roles and
\end{abstract}

S. Cook

Cancer Care Nova Scotia, Halifax, Canada

e-mail: sandra.cook@ccns.nshealth.ca

\author{
A. F. Howard $(\bowtie) \cdot$ K. Smillie $\cdot$ V. Chan $\cdot$ A. Kazanjian \\ School of Population and Public Health, Faculty of Medicine, \\ University of British Columbia, 2206 East Mall, Vancouver, BC V6T \\ $1 \mathrm{Z3}$, Canada \\ e-mail: fuchsia.howard@ubc.ca \\ K. Smillie \\ e-mail: kirsten.smillie@ubc.ca \\ V. Chan \\ e-mail: vwychan@interchange.ubc.ca \\ A. Kazanjian \\ e-mail: arminee.kazanjian@ubc.ca
}

responsibilities also reflected local context in their approaches to patient assessment, referral, education, coordination of services, and advocacy. Third, these two distinct approaches to navigation have responded to the needs of diverse populations being served by improving access to care.

Conclusions Evidence generated using the KE-DS Model could ensure a more robust and structured approach to the planning and implementation of future navigation programs. The Model prompts users to make explicit the different types of evidence utilized during program planning and implementation. The systematic collection of new information on program implementation using the KE-DS Model in future initiatives will contribute to an improved understanding of the science of knowledge exchange.

Keywords Knowledge exchange · Patient navigation · Supportive cancer care

\section{Abbreviations}

KE

KE-DS Knowledge-exchange decision support

Model model

\section{Background}

The exchange of information and evidence has been recognized as critical to promoting evidence-informed best practices in health care [1]. Traditionally, knowledge exchange was viewed as linear and unidirectional, whereby researchers generate knowledge that can be disseminated and adopted by clinicians and policy makers. This conceptualization does not accurately depict the dynamic and complex set of relationships between knowledge producers and users nor does it recognize the 
importance of adapting knowledge to the local setting [2, 3]. While evidence-informed practice has been a widespread expectation for a number of years, the persistent gap between knowledge production and use is also well recognized [4].

Models that highlight the dialogical exchange of knowledge between knowledge producers and users have been identified as more effective approaches to knowledge exchange [5]. For researchers to translate evidence into a meaningful product and for knowledge users to inform the research agenda so that meaningful questions are investigated, interaction at all stages of the research process is the key $[6,7]$. There is a gap in the implementation science literature concerning evidence-informed health care program implementation strategies [8] and factors that contribute to program sustainability $[9,10]$.

Recognizing the importance of collaboration, we designed the Knowledge Exchange-Decision Support (KE-DS) Model to animate the producer/user dynamic and facilitate the exchange of expertise and evidence. The KE-DS Model provides a framework outlining the essential domains of knowledge generation and exchange. The KE-DS Model shows the iterative set of interactions between the creation of new information, strategies for dissemination and translation, and opportunities related to implementation of new knowledge into current practice. The KE-DS Model reflects the principles of evidence-informed medicine and was tailored specifically for cancer survivorship programs [11-13].

The KE-DS Model considers the traditional Health Technology Assessment dimensions $[14,15]$ but also teases out the epidemiological, social and economic contexts, and the source, nature and quality of evidence. The Model prompts the structured exploration of each of these domains. The population of interest, an epidemiological construct, is defined by the sociodemographic, geographic, and cultural attributes of the people targeted by the intervention or program. In this Model, the social and economic scope of the program is examined at the individual, community, and organizational levels. Evidence includes scientific research and experiential knowledge recognizing that both producers and end users of knowledge draw from a diversity of knowledge forms acquired over a period of time [2, 16-18].

Patient navigation has been proposed to improve access to appropriate supportive care and enhance cancer survivorship. The navigator role has been used "to denote a system or professional role primarily intended to expedite patient access to services and resources and improve continuity and coordination of care throughout the cancer continuum" [19]. The concept was first developed and tested in the USA by Dr. Harold P. Freeman [20] as a means to assist low income women in accessing screening and treatment for breast cancer and was associated with improvements in diagnosis outcomes [21]. While different models of patient navigation have been studied since then, there is no consensus on the best approach
$[22,23]$. Navigation programs have been piloted by the Northern Health Authority in the Western province of British Columbia and in the Eastern province of Nova Scotia by Cancer Care Nova Scotia. The purpose of the research described here is to illustrate how the KE-DS Model makes explicit the specific approaches in the planning and implementation of two navigation programs in Canada that otherwise are assumed to be uniform and to delineate the $\mathrm{KE}$ interactions occurring during these processes.

\section{Methods and data collection}

Data was collected that described the development and implementation of the navigation programs in Northern British Columbia and across Nova Scotia. The collection and subsequent qualitative thematic analysis of this data was guided by the KE-DS Model. The participants in this research are the leads and staff of two navigation programs in Canada.

The KE-DS research team initially introduced the Model to the navigation program teams at in person meetings. In Northern British Columbia, the KE-DS Model was applied at the inception of the navigation program. Subsequent communication included phone calls and e-mails throughout the navigation project implementation. In Nova Scotia, the KE-DS Model was used after the implementation of the navigator program. The navigation program team was instructed on how to apply the KE-DS Model to their program. This was followed by a site visit.

Detailed descriptions were gathered that captured the nuances and subtleties of navigation program planning and implementation at these two navigation sites. These descriptions included detailed notes stemming from interviews, observations, informal correspondence, e-mail exchanges between research and project teams, and end of project phone interviews between the KE-DS research team and navigation project leads. During interviews, we posed closed- and openended questions related to each step in the KE-DS Model (see Table 1). Throughout data collection, the KE-DS research team verified notes through e-mail with navigation project leads who contributed comments, clarified details, and elaborated on the processes involved in planning and implementing the project. We collected data during a 16-month work period.

\section{Data analysis}

During the analysis of data, we coded site specific notes according to the steps of the KE-DS Model (see Table 1), treating each of these steps as a theme. These were establishing the need, site description, stakeholders, population of interest, population impact, social context, and economic context. We then prepared descriptive summaries for each navigation 
Table 1 Closed- and open-ended questions related to each step in the KE-DS Model

\begin{tabular}{l}
$\begin{array}{l}\text { KE-DS Model guiding Action } \\
\text { steps }\end{array}$ \\
\hline
\end{tabular}

Summarize the intervention

Establish current literature and evidence to consider

Identify stakeholders

Consider population context

Identify program specific population of interest

Identify program specific population impact

Quantify health concerns using indicators

Consider economic context Assess economic concerns and variables at individual, community, and organization levels.

\section{Consider social context \\ Revisit the intervention: evaluate how much and for who \\ Strategies for dissemination}

Document resources, tools and reports in the current literature, experiential knowledge, etc. that support the choice of the program
Assess the social scope at an individual, community, and organizational level

Assess the intervention in light of all contextual issues.

Identify all of the end users of the newly framed knowledge and understanding of the program or intervention.
- Delineate what is known from existing research.

- Consider the research evidence alongside ethical concerns, the sociocultural norms of end users, and specific health systems.

- Delineate what is known from clinical experience.

- Delineate what is the experiential and cultural knowledge.

- Are the interventions or supportive care services identified as best practices or evidence based?

- What is the merit/value of the evidence/technology?

-What is the impact value of evidence for stakeholders?

- Does your site have a broad-based, balanced stakeholder group that includes people from each of the suggested groups?

- Are these individuals available and willing to contribute to the planning and development processes?

- Are there a decision-making body/team, project timelines, communication strategies, and logistical plans in place?

- Who are the people you are targeting with the research, program, or initiative? Who is the "population at risk?"

- Define the groups using sociodemographic, geographic, and/or ethnic/cultural factors.

- Confirm that this population group is the focus of the current priority in macro and micro Health Care environments.

- Consider the logistical issues of targeting this population - what are the challenges you might encounter? What are the supports?

- Quantify health concerns using indicators based on the natural history of the disease, the size of the population, the ability to access the population etc.

-What do you hope to accomplish for the population of interest?

- Describe anticipated population impact in terms of population health and health systems research.

-What are relevant measures to gauge improvement?

- What is appropriate and relevant program, process, or intermediate outcome measures?

- Unit costs versus total costs, direct, direct non-health, indirect, and intangible care costs.

- Potential costs to the individual, community, and organization, and impact on other services and supporting groups.

- Medical cost offset

- Outcome measures: future use of services

- Power/status and dominance issues

- Personal/public values

- People's perspectives that will influence the success of the program

- Consider documented and experiential evidence in order to confirm goals and uptake.

-What strategies will be used to engage them?

- At what point during the work will these strategies be employed? project site according to these themes. Summaries were then compared and contrasted noting similarities and differences throughout the process of planning and implementing the navigation programs.

\section{Results}

Northern British Columbia and Nova Scotia differed in how navigation was conceptualized and adapted to meet their 
community needs. Using the KE-DS Model as a framework, we highlighted a variety of contextual aspects that shaped planning and delivery of these navigation programs. Here, we describe the three factors that were most influential for participants at both sites: the structure of health services, professional roles and responsibilities, and the population being served.

\section{The structure of health services}

The KE-DS Model prompts program teams to explore and articulate who the stakeholders are and how the organizations or systems that they operate within can participate in and may influence program planning and implementation. Through data analysis guided by the KE-DS Model, the structure of health services was among the most important factors in the contexts examined. In both Northern British Columbia and Nova Scotia, the structure of health services is largely defined by the interaction of professionals and services operating within that jurisdiction. The structure of health services in Northern British Columbia and Nova Scotia reflects their respective geographic environments. Both study sites serve rural communities with varying degrees of remoteness and challenges accessing services. Framed as a strategy to improve access to services and continuity of care, both navigation programs were supported by their respective health authorities and provincial governments. These navigation programs were only possible because of buy-in from decision makers and stakeholders throughout the health authorities. For example, in Northern British Columbia, the Northern Health Board and the Chief Nursing Officer endorsed the navigation program. Buy-in from stakeholders is an important factor for program feasibility highlighted by the KE-DS Model. The Model prompts users to consider to what degree individuals are available and willing to contribute to the planning and development processes.

Northern British Columbia designed their navigation program to focus on improving systems to guide patients through trajectories of care. A system approach to improve health services delivery was deemed more appropriate because of the limited health human resources in the large Northern region and the inability to achieve a one-to-one navigator-topatient ratio. The focus is not only assessing what types of services are required to address patient and family needs but also how the existing services are organized to provide coverage across the region. This approach is aimed at leveraging human resources by coordinating multiple health services in that region. This became apparent through use of the Model as it directs users to consider, in addition to individuals, organizations and systems as stakeholders.

The KE-DS Model prompts the consideration of relevant evidence, including scientific evidence, organizational documents (such as financial and program reports), and experiential knowledge of clinicians, administrators, patients, and their families. KE-DS questions related to knowledge and evidence include: What is known from clinical experience?; Are the interventions of supportive care services identified as best practices or evidence based?; and What is the impact or value of evidence for stakeholders? As the Northern British Columbia navigation program was one of the first attempts to approach navigation from a systems level, that is, to address system wide problems of mix and distribution of human resources, there was limited research evidence to draw upon. Implementation was not without its challenges. When interviewed, the Northern British Columbia navigator reported that she works with health care providers to promote a shared understanding of the entire patient journey and to coordinate care. She commented that this novel and unfamiliar approach led to uncertainty about whether they were "doing the right thing" even though this approach was chosen specifically with the structure of health services in mind. She further commented that "hesitation was the biggest obstacle" among health care providers. Getting to know the structure of health services and the many different service providers throughout the North was initially demanding for the newly appointed navigator. Furthermore, maintaining relationships with stakeholders, including health care providers, continues to require substantial time and effort on behalf of the navigator despite having received the support from many stakeholders.

Similar to Northern British Columbia, in Nova Scotia, the experiential knowledge of patients and health care professionals was also of utmost importance in selecting a nurseled approach. A needs assessment with community members and clinicians was the foundation for their navigation program. Navigation was identified as an intervention to improve cancer care for individuals. The navigator role in Nova Scotia follows a one-to-one, nurse-led model where the patient is followed throughout the cancer trajectory from the time of diagnosis through to end of treatment and survivorship. This approach is informed by a case management model and provides a focal point for entry into the cancer system. The navigators work in districts without a cancer center and have responsibility for cancer patients across these districts. One of the two provincial cancer centers refers discharged patients to the community navigators in their district.

The integration of the Nova Scotia navigator role into existing health services posed unique challenges. The KEDS Model guides users to assess the social context at the individual, community, and organizational level. These components make explicit power, status and dominance issues, and personal, professional, and public values and perspectives that could influence the success of the program. When interviewed, the navigation manager stressed the importance of professional title in shaping how the navigators are perceived by other health care providers. Initially, other clinicians were skeptical even though the role is required to be filled by 
an experienced registered nurse. The navigators reported that the title "navigator" does not sound like a medical/clinical role, and that the majority of the programs in navigation at the time were in the USA and primarily led by volunteers and peers and were intended to reduce disparities in underserved populations. The navigation manager (SC) stated that in hindsight, she would not use the title navigator to identify this role. At the site visit in Nova Scotia, the navigators described how some health care providers expressed concern that this new role would negatively influence their own positions and questioned "who is being fired if a new position is being developed?" The navigators reported that some health care professionals were defensive and of the opinion that they already provided this service. These reactions suggest that health care professionals are aware of the costs of health services and the importance and implications of fiscal responsibility on behalf of institutions, as well as an element of professional territoriality.

\section{Professional roles and responsibilities}

When applying the KE-DS Model to Northern British Columbia and Nova Scotia navigation programs, the professional roles and responsibilities of the navigator greatly influenced program planning and implementation, but also, these roles and responsibilities evolved through the development of the navigation programs to meet community needs. When viewed through the lens of the Model, the professional roles and responsibilities of both programs were largely defined by the economic context, that is, fiscal constraint and the social context of rural and diverse communities.

In Northern British Columbia, the main role of the navigator is to coordinate care. The navigator is located in the main urban center that patients travel to for medical care. The Northern British Columbia navigator works primarily with hospital staff and health care professionals to coordinate care. This involves bringing various clinicians together to encourage communication and promote a shared understanding of the entire patient journey. While the navigator's focus is services delivered in the main urban centre, she communicates weekly with the dietician, social worker, and clinical educator who serve patients in outlying areas. Education and advocacy are the navigator's responsibility in the North. The navigator focuses on developing and distributing educational packages for breast and colorectal cancer patients rather than one-on-one teaching. The standardized packages reflect population needs and the resources available in the North. In addition, the navigator also educates health care professionals in her own and surrounding communities about available resources and services. The navigator also plays an important role in identifying gaps in health services and advocating for appropriate services. For example, the navigator facilitated the development of a comprehensive breast clinic that provides screening, diagnostic testing, pre- and postsurgical care, patient education, and support services.

The professional roles and responsibilities associated with a one-to-one, nurse-led approach to navigation are diverse and vary according to the institution or community where they operate. Nurse-led navigation, however, often includes patient assessment, referral, education, and coordination of services. In Nova Scotia, the navigator sees the patient at all of the critical times in their cancer journey. Much of the navigator role is to screen and assess patients for clinical and supportive care needs: provide consistent education and information to patients and families, coordinate care between health settings and refer patients to the most appropriate services, provide emotional support and work with patients to overcome logistical challenges. These navigators do not provide medical treatments. The one-on-one approach allows the navigator to assess patients within the context of their lives, their cultural beliefs, situational context, the availability of social support, and access to transportation. As in Northern British Columbia, patient education is also a main function of the role, wherein the navigators provide and reinforce education received from members of the health care team. They also book appointments and coordinate services that facilitate continuity of care. For example, if a patient is traveling a long distance, the navigator can assist in scheduling all of their appointments on the same day. These community immersed navigators identify patient needs and gaps in services and advocate for patients by informing system managers/officials of services that are needed in their area, including structural barriers. For example, one navigator was reported to be instrumental in revising an outdated policy prohibiting the use of complementary medicine.

Application of the KE-DS Model enabled the research team to compare and contrast the structural characteristics within which the two navigation programs were delivered and to highlight how the professional practices of the navigators responded to the needs of their respective communities. The KE-DS Model helped illuminate how in both Northern British Columbia and Nova Scotia's professional roles and responsibilities evolved based on a thorough understanding of existing resources, gaps in services, and the needs of cancer survivors. From the perspective of the KE-DS research team, this contributed to program success and sustainability.

\section{Population being served}

The KE-DS Model prompts project teams to explore and articulate the epidemiological dimensions regarding population at risk and population impact to gauge program effect at the population level. What are the challenges and supports that might be encountered in targeting the group with most need? 
The KE-DS research team asked the project teams to identify the people targeted with each navigation program and to define the population at risk. In Northern British Columbia, the navigation program has addressed service needs for those accessing care in the most populated or urban area of this health region. According to the navigator, the number of patients accessing services and resources increased shortly following the implementation of the navigation program. Moreover, while initially intended to address services for breast cancer patients, the navigation program has had unintended benefits for others, such as those with colorectal cancer. The population of interest has now grown to include all cancer patients accessing care in the North. The KE-DS Model prompts users to revisit the program objectives and evaluate whose needs are being met, and of equal importance, whose needs are not met. The Northern British Columbia navigator expressed concern that the smaller more remote communities that do not have services under the jurisdiction of a navigator may not have benefited from systems level changes to Northern Health services. These communities include aboriginal and non-aboriginal individuals.

When reviewing the program through the lens of the KEDS Model, it became apparent that the Nova Scotia navigation program was receptive to very specific population needs. The navigators reported that being embedded in the local community was the key to program success as they learned about the culture of the community, the services available, and population needs. Nova Scotia has a diverse population that includes large First Nations, African- and Arab-Canadian populations. Since the inception of the navigator role, effort has been made to meet the needs of diverse, ethno-cultural communities which are not being met. For example, a needs assessment was conducted with First Nations, African Nova Scotian and immigrant women. A dedicated staff person familiar with these communities now works with the navigators to develop strategies to address specific community challenges. Thus far, a number of projects have begun, such as peer education and the development of an immigrant women's health clinic.

Despite differences between Northern British Columbia and Nova Scotia, patients in both provinces experience common challenges accessing care stemming from the rural environment and weather conditions. "The challenges imposed by long distances and harsh weather add to the problems faced by northern patients who need to travel to access specialists, screening services, diagnostic tests, and treatment. The full range of services is often not available in one location thus requiring patients to travel to multiple locations at multiple times" (The Northern Cancer Control Strategy Business Plan, 2009). In Northern British Columbia and Nova Scotia, the navigation programs have improved access by decreasing travel and the associated costs. For example, while the Northern British Columbia navigator did not deliberately consider navigation as an intervention to reduce patient costs, the
Northern breast clinic eliminated the need for breast cancer patients to make multiple visits for screening and diagnostics.

The KE-DS Model prompted program planners to evaluate population impact and long-term goals. Specific questions included: what do you hope to accomplish for the population of interest, and what are relevant measures to gauge improvement? Means of assessing improvements in patient outcomes associated with the implementation of the navigation program have evolved since the planning stage. The Northern British Columbia navigator initially expressed difficulty finding measures to evaluate broader goals when prompted by the KE-DS Model, therefore, were reliant on intermediate or proxy outcome measures. They expected to rely on patient surveys to assess the impact of the navigation program. Improving patient throughput was considered a measure to evaluate achievement; however, the navigation program considered obtaining patients' perspectives and experiences to be the most important. Once the navigator program was underway, the navigator developed a short questionnaire to obtain patient feedback. Examples of questions included, "Did you at any time feel as though you did not have a support system behind you?" and "Did you ever feel lost?" Information gathered through this questionnaire is currently informing refinement of cancer services. In Nova Scotia, the navigation manager reported that feedback from other clinicians and patients has been extremely positive. For example, one oncologist stated that they would prefer to only see patients in communities that are served by a navigator. More specific patient outcome evaluations are currently under development.

\section{Discussion}

This research is a demonstration of how the KE-DS Model was applied to practice environments. We describe the planning and implementation of two approaches to navigation in cancer care through the lens of this knowledge exchange model, the KE-DS Model. These two approaches included a systems level approach implemented in Northern British Columbia and a one-to-one, nurse-led model in Nova Scotia. The KE-DS Model explicated contextual factors important to the planning and implementation phases of these navigation programs. The three most pertinent factors elicited in both programs were the structure of health services, professional roles and responsibilities, and the population being served.

As used by these two program teams, the emphasis of the KE-DS Model is more on making explicit what constitutes information and knowledge than the translation of established evidence. Therefore, this Model is well suited for real-world clinical and community settings where a problem is identified for intervention but the research evidence available is not contextualized to the specific setting. While the strengths of existing models such as the Knowledge to Action framework 
and the Ottawa Model for Research Use [24, 25] lie in their applicability to instances where high quality research evidence exists is highly generalizable and can form the base unit for specific knowledge translation activities, the KE-DS Model is useful in common situations where decision makers and clinicians are challenged to improve patient care despite a dearth of applicable research evidence. Various forms of knowledge are recognized as integral but are often taken for granted. The Model offers users a method of documenting, organizing, and summarizing these different forms of knowledge into a format that is easier to appreciate and utilize.

The tailoring of knowledge, research evidence, and programmatic experience to meet individual or community needs is a primary obstacle in health care delivery, yet this is essential if knowledge, evidence, and interventions are to serve the public interest. Research evidence in support of navigation was important for initially choosing navigation as an intervention. However, the implementation of navigation was largely shaped by structural (organizational, professional, and social) factors of the two communities. No two communities are identical, but lessons learned in one community could provide insight for other communities that have similar needs and interests in implementing interventions such as navigation.

In addition to a systems level approach and a nurse-led approach, electronic pathways and virtual navigation have also been explored [26]. In light of inconclusive evidence and a lack of consensus regarding the impact of different navigation models, the current research provides support for those advocating for assessing the needs of the populations served and tailoring navigation interventions accordingly [27]. However, it is also worth noting the opposition to the creation of an additional navigator role in the specific Canadian context [23].

Over the past 20 years, a number of models to explain and promote evidence-based practice have emerged. The emphasis of the majority of these models has been on the importance of translating evidence into clinical practice by changing the behavior of health care professionals. Changing clinical practice alone, however, is not sufficient for improving care planning and delivery [25]. Attention to knowledge exchange strategies at the organizational or systems level is also necessary to support and maintain changes in practitioner behavior. This research exemplifies the utility of the KE-DS Model to bring out multiple perspectives (e.g., the role of social context) and forms of knowledge in health service planning and delivery.

As directed by the KE-DS Model, program planning and implementation was tailored to the needs of specific end users in Northern British Columbia because stakeholders were engaged throughout the process. This is similar to previous research that suggests knowledge exchange strategies are more effective and achievable when a broad complement of stakeholders are involved [28]. However, while patient and community members were consulted in the beginning of program planning and implementation in both Northern British Columbia and Nova Scotia, there was no formal means of consistently engaging patients and families throughout the implementation and evaluation phases. Although the importance of involving all stakeholder groups is subsumed within the KE-DS Model, it perhaps requires greater emphasis and attention with project teams in the initial planning stages so that patient and family engagement strategies are explicitly established and continued.

Evaluations must demonstrate the effectiveness of programs for continued stakeholder support. While both the Northern British Columbia and Nova Scotia navigators were aware of the importance of evaluation for sustaining these programs and demonstrating their relevance, feasibility, and impact, standardized evaluations for these different navigation models have yet to be developed. As a result, those implementing navigation face the challenge of developing evaluation tools, a challenge identified by others working in the area of navigation [22, 23]. Although this presents a barrier to program refinement, this is an opportunity to develop evaluations that will reflect context relevant outcomes.

\section{Conclusions}

The KE-DS Model provides a tool for incorporating diverse forms of knowledge into health services planning and delivery. The implementation of navigation programs in Canada and internationally will likely continue. Evidence generated using the KE-DS Model, as shown here, could ensure a more robust and structured approach to the planning and implementation of navigation programs. The systematic collection of new knowledge using the KE-DS Model in future initiatives will contribute to an improved understanding of the science of knowledge exchange.

Acknowledgments This study was made possible by funding from the Canadian Institute of Health Research (no. AQC83559) and the Canadian Partnership Against Cancer. Dr. Fuchsia Howard holds a Michael Smith Foundation for Health Research (MSFHR) Postdoctoral Research Trainee Award.

Conflict of interests We have no conflict of interest.

Open Access This article is distributed under the terms of the Creative Commons Attribution Noncommercial License which permits any noncommercial use, distribution, and reproduction in any medium, provided the original author(s) and the source are credited.

\section{References}

1. Satterfield J, Spring B, Brownson R, Mullen E, Newhouse R, Walker B, Whitlock E (2009) Toward a transdisciplinary model of evidencebased practice. Milbank Q 87(2):368-390. doi:10.1111/j.1468-0009. 2009.00561.x 
2. Baumbusch JL, Kirkham SR, Khan KB, McDonald H, Semeniuk P, Tan E, Anderson JM (2008) Pursuing common agendas: a collaborative model for knowledge translation between research and practice in clinical settings. Res Nurs Health 31:130-140. doi:10.1002/nur. 20242

3. Graham I, Logan J, Harrison M, Straus S, Tetroe J, Caswell W, Robinson-Goldenberg N (2006) Lost in knowledge translation: time for a map? J Contin Educ Health Prof 26:13-24. doi:10.1186/17485908-7-44

4. Ward V, House A, Hammer S (2009) Knowledge brokering: exploring the process of transferring knowledge into action. BMC Health Serv Res 9:12. doi:10.1186/1472-6963-9-12

5. Canadian Institute of Health Research. Knowledge Translation Strategy 2004-2009 Innovation in Action. Canada, Available: http://www.cihr-irsc.gc.ca/e/26574.html. Accessed 6 Dec 2012

6. Lavis J (2006) Research, public policymaking, and knowledgetranslation processes: Canadian efforts to build bridges. J Contin Educ Health Prof 26:37-45. doi:10.1002/chp.49

7. Ross S, Lavis J, Rodriguez C, Woodside J, Denis J (2003) Partnership experiences: involving decision makers in the research process. J Health Serv Res Policy 8:26-34. doi:10.1258/ 135581903322405144

8. Lavis J, Ross S, Hurley J, Hohenadel J, Stoddart G, Woodward C, Abelson J (2002) Examining the role of health services research in policy making. Milbank Q 80(1):125-154. doi:10.1111/1468-0009.00005

9. Greenhalgh T, Macfarlane R, Bate P, Kyriakidou O (2004) Diffusion of innovation in service organizations: systematic review and recommendations. Milbank Q 82(4):581-629. doi:10.1111/j.0887-378X. 2004.00325.x

10. Wiltsey-Stirman S, Kimberly J, Cook N, Calloway A, Castro F, Charns M (2012) The sustainability of new programs and innovations: a review of the empirical literature and recommendations for future research. Implement Sci 7:17. doi:10.1186/1748-5908-7-17

11. Kazanjian A, Smillie K, Howard AF, Ward A, Doll R (2012) A structured approach to knowledge exchange: understanding the implementation of a cancer survivor program. Eur J Oncol Nurs 16(4): 399-405. doi:10.1007/s00520-012-1686-2

12. Kazanjian A, Howett C, Chan V, Smillie K (2009a) Knowledge exchange decision-support toolkit for supportive cancer care, final report 2009. http://spph.ubc.ca/sites/healthcare/files/2009/KE-DS_ Toolkit October2009.pdf Accessed on 6 Dec 2012

13. Kazanjian A, Howett C, Smillie K (2009b) Supportive cancer care programs: further application of the knowledge exchange-decision support toolkit final report, 2009. http://spph.ubc.ca/sites/healthcare/files/ 2009/KEDS_Report_Cancer_Transitions_OCT09.pdf Accessed on 6 Dec 2012

14. Banta HD, Luce BR (1993) Health care technology and it's assessment: an international perspective. Oxford University, New York
15. Kazanjian A (2004) Reflections on the social epidemiological dimension of health technology assessment. Int J Technol Assess Health Care 20(2):167-173. doi:10.1017/S0266462304000947

16. Bartunek J, Trullen J, Bonet E, Sauquet A (2003) Sharing and expanding academic and practitioner knowledge in health care. J Health Serv Res Policy 8(2):62-68. doi:10.1136/bmjqs.2010. 046169

17. Goldenberg MJ (2006) On evidence and evidence-based medicine: lessons from the philosophy of science. Soc Sci Med 62:2621-2632. doi:10.1016/j.socscimed.2005.11.031

18. Lambert H (2006) Accounting for EBM: notions of evidence in medicine. Soc Sci Med 62:2633-2645. doi:10.1016/j.socscimed. 2005.11.023

19. Sociobehavioural Research Centre, BC Cancer Agency (2005) Patient navigation in cancer care. Final report. Vancouver, Canada http://www.bccancer.bc.ca/NR/rdonlyres/FBBD2D27-7E88-4340AE76-5552E2B57C5C/14927/print_Final_Navigation1.pdf. Accessed on 9 Sep 2013

20. Freeman HP, Muth BJ, Kerner JF (1995) Expanding access to cancer screening and clinical follow-up among the medically underserved. Cancer Pract 3(1): 19-30

21. Oluwole SF, Ali AO, Adu A, Blane BP, Barlow B, Oropeza R, Freeman HP (2003) Impact of a cancer screening program on breast cancer stage at diagnosis in a medically underserved urban community. J Am Coll Surg 196:180-188. doi:10.1016/S1072-7515(02)01765-9

22. Wells K, Battaglia T, Dudley D, Garcia R, Greene A, Calhoun E (2008) Patient navigation: state of the art or is it science? Cancer 113(8):1999-2010. doi:10.1002/cncr.23815

23. Thorne S, Truant T (2010) Will designated patient navigators fix the problem? Oncology nursing in transition. Can Oncol Nurs J20(3): $116-128$

24. Logan J, Graham ID (1998) Toward a comprehensive interdisciplinary model of health care research use. Sci Commun 20:227-246. doi: $10.1177 / 1075547098020002004$

25. Straus S, Tetroe J, Graham I (2009) Knowledge translation in health care: moving from evidence to practice. Blackwell Publishing, Oxford

26. Loiselle C, Peters O, Haase KR, Girouard L, Korner A, Wiljer D, Fitch M (2013) Virtual navigation in colorectal cancer and melanoma: an exploration of patients' views. Support Care Cancer 21(8): 2289-2296. doi:10.1007/s00520-013-1771-1

27. Paskett ED, Harrop JP, Wells KJ (2011) Patient navigation: an update on the state of the science. CA Cancer J Clin 61(4):237-249. doi:10. $3322 /$ caac. 20111

28. Gagliardi A, Fraser N, Wright F, Lemieux-Charles L, Davis D (2008) Fostering knowledge exchange between researchers and decisionmakers: exploring the effectiveness of a mixed-methods approach. Health Policy 86:53-63. doi:10.1016/j.healthpol.2007.09.002 